PROCEEDINGS OF THE

AMERICAN MATHEMATICAL SOCIETY

Volume 134, Number 11, November 2006, Pages 3363-3372

S 0002-9939(06)08368-7

Article electronically published on May 18, 2006

\title{
UNIQUENESS IMPLIES EXISTENCE AND UNIQUENESS CRITERION FOR NONLOCAL BOUNDARY VALUE PROBLEMS FOR THIRD ORDER DIFFERENTIAL EQUATIONS
}

\author{
STEPHEN CLARK AND JOHNNY HENDERSON \\ (Communicated by Carmen C. Chicone)
}

\begin{abstract}
For the third order differential equation, $y^{\prime \prime \prime}=f\left(x, y, y^{\prime}, y^{\prime \prime}\right)$, we consider uniqueness implies existence results for solutions satisfying the nonlocal 4-point boundary conditions, $y\left(x_{1}\right)=y_{1}, y\left(x_{2}\right)=y_{2}, y\left(x_{3}\right)-y\left(x_{4}\right)=y_{3}$. Uniqueness of solutions of such boundary value problems is intimately related to solutions of the third order equation satisfying certain nonlocal 3-point boundary conditions. These relationships are investigated as well.
\end{abstract}

\section{Introduction}

In this paper, we are concerned with uniqueness and existence of solutions of certain types of boundary value problems for third order differential equations. In particular, we deal with uniqueness implies existence questions for solutions of the third order ordinary differential equation,

$$
y^{\prime \prime \prime}=f\left(x, y, y^{\prime}, y^{\prime \prime}\right), \quad a<x<b,
$$

satisfying nonlocal 4-point boundary conditions given by

$$
\begin{aligned}
& y\left(x_{1}\right)=y_{1}, \quad y\left(x_{2}\right)=y_{2}, \quad y\left(x_{3}\right)-y\left(x_{4}\right)=y_{3}, \\
& y\left(x_{1}\right)-y\left(x_{2}\right)=y_{1}, \quad y\left(x_{3}\right)=y_{2}, \quad y\left(x_{4}\right)=y_{3},
\end{aligned}
$$

where $a<x_{1}<x_{2}<x_{3}<x_{4}<b$ and $y_{1}, y_{2}, y_{3} \in \mathbb{R}$. We also consider solutions of (1.1) satisfying nonlocal 3 -point boundary conditions given by

$$
\begin{aligned}
& y\left(x_{1}\right)=y_{1}, \quad y^{\prime}\left(x_{1}\right)=y_{2}, \quad y\left(x_{2}\right)-y\left(x_{3}\right)=y_{3}, \\
& y\left(x_{1}\right)-y\left(x_{2}\right)=y_{1}, \quad y\left(x_{3}\right)=y_{2}, \quad y^{\prime}\left(x_{3}\right)=y_{3},
\end{aligned}
$$

where $a<x_{1}<x_{2}<x_{3}<b$ and $y_{1}, y_{2}, y_{3} \in \mathbb{R}$.

Remark 1.1. For the remainder of this paper, we shall denote the boundary value problem consisting of equation (i) and boundary condition (j) by (i):(j).

Received by the editors February 18, 2005 and, in revised form, May 20, 2005 and June 11, 2005.

2000 Mathematics Subject Classification. Primary 34B15; Secondary 34B10.

Key words and phrases. Boundary value problem, uniqueness, existence, nonlocal.

Research for the first author was partially supported by NSF Grant DMS-0405528, as well as by a Baylor University Visiting Professorship during the Fall of 2004.

(C)2006 American Mathematical Society Reverts to public domain 28 years from publication 
Interest in multipoint boundary value problems for lower order differential equations has been ongoing for several years. For a small sample of such work, we refer the reader to papers by Bai and Fang [1, Gupta and Trofimchuk [3] and Ma [20, 21].

Questions of the type with which we deal in this paper have been considered for solutions of (1.1) satisfying 3-point conjugate boundary conditions given by

$$
y\left(x_{1}\right)=y_{1}, \quad y\left(x_{2}\right)=y_{2}, \quad y\left(x_{3}\right)=y_{3},
$$

and 2-point conjugate boundary conditions given by

$$
\begin{aligned}
& y\left(x_{1}\right)=y_{1}, \quad y^{\prime}\left(x_{1}\right)=y_{2}, \quad y\left(x_{2}\right)=y_{3}, \\
& y\left(x_{1}\right)=y_{1}, \quad y\left(x_{2}\right)=y_{2}, \quad y^{\prime}\left(x_{2}\right)=y_{3},
\end{aligned}
$$

where $a<x_{1}<x_{2}<x_{3}<b$, and $y_{1}, y_{2}, y_{3} \in \mathbb{R}$. These questions have involved (i) whether uniqueness of solutions of (1.1):(1.6) implies uniqueness of solutions for (1.1):(1.7) and (1.1):(1.8); (ii) whether uniqueness of solutions of (1.1):(1.7) and (1.1):(1.8) implies uniqueness of solutions of (1.1):(1.6); and (iii) whether uniqueness of solutions of (1.1):(1.6) implies the existence of solutions of (1.1):(1.6), (1.1):(1.7), and (1.1):(1.8). Of course, a principal reason for considering questions such as (i) or (ii) would be in resolving question (iii).

Hypothesis 1.2. With respect to (1.1), we assume throughout that

(A) $f\left(x, r_{1}, r_{2}, r_{3}\right):(a, b) \times \mathbb{R}^{3} \rightarrow \mathbb{R}$ is continuous;

(B) solutions of initial value problems for (1.1) are unique and extend to $(a, b)$.

Given this hypothesis, and in a context of nth order problems, Jackson 14 provided an affirmative answer to (i). In a like manner, Jackson [15] gave an affirmative answer to question (ii). Finally, for question (iii), the paper by Jackson and Schrader [16], in conjunction with papers for $n t h$ order problems by Hartman 4, 5] and Klaasen [17, provides a positive answer.

There have been other works devoted to uniqueness questions of these types as well as uniqueness implies existence questions for boundary value problems. These works have often been for $n t h$ order problems for ordinary differential equations, for finite difference equations, and recently for dynamic equations on time scales; see the extensive list of references in [2], 6] - [13, [18, [19] and [22.

In this paper, we consider analogues of questions (i)-(iii) relative to the boundary value problems (1.1):(1.2), (1.1):(1.3), (1.1):(1.4), and (1.1):(1.5). Section 2 is devoted to uniqueness implies uniqueness relationships among these boundary value problems, whereas Section 3 will center on uniqueness implies existence results for the same boundary value problems.

\section{UNIQUENESS OF SOLUTIONS}

Given Hypothesis 1.2, we now show that uniqueness of solutions for (1.1):(1.2) and (1.1):(1.3) implies the uniqueness of solutions for (1.1):(1.4) and (1.1):(1.5), and conversely.

Remark 2.1. (a) If solutions of (1.1):(1.2) or (1.1):(1.3) are unique when they exist, then solutions of the 3-point conjugate problems (1.1):(1.6) are unique. To see this, suppose solutions of (1.1):(1.2) are unique, but that for some $a<x_{1}<x_{2}<x_{3}<b$, there exist distinct solutions, $y$ and $z$ of (1.1) so that $y\left(x_{i}\right)=z\left(x_{i}\right)$, for $i=1,2,3$. Define $w:=y-z$ and suppose, without any loss of generality, that $w(x)>0$ on 
$\left(x_{2}, x_{3}\right)$. Then, $w(x)$ has a positive maximum in $\left(x_{2}, x_{3}\right)$, and there exist points $x_{2}<\tau_{1}<\tau_{2}<x_{3}$ so that $w\left(\tau_{1}\right)=w\left(\tau_{2}\right)$. Then, for $x_{1}<x_{2}<\tau_{1}<\tau_{2}$, we have $y\left(x_{1}\right)=z\left(x_{1}\right), y\left(x_{2}\right)=z\left(x_{2}\right), y\left(\tau_{1}\right)-y\left(\tau_{2}\right)=z\left(\tau_{1}\right)-z\left(\tau_{2}\right)$, which contradicts the uniqueness of solutions of (1.1):(1.2). As a consequence of statements in the Introduction, each of the conjugate boundary value problems (1.1):(1.6), (1.1):(1.7) and (1.1):(1.8) has a unique solution.

(b) If solutions of (1.1):(1.4) and (1.1):(1.5) are unique, when they exist, then solutions of the 2-point conjugate problems (1.1):(1.7) and (1.1):(1.8) are unique. Again, from statements in the Introduction, each of the conjugate boundary value problems (1.1):(1.6), (1.1):(1.7) and (1.1):(1.8) has a unique solution.

Our first result of this section will deal with continuous dependence of solutions of (1.1) on boundary conditions.

Theorem 2.2. Assume that solutions of (1.1):(1.2) are unique, when they exist. Given a solution $y(x)$ of (1.1) on $(a, b)$, an interval $[c, d] \subset(a, b)$, points $c<x_{1}<$ $x_{2}<x_{3}<x_{4}<d$, and an $\epsilon>0$, there exists a $\delta(\epsilon,[c, d])>0$ such that, if $\left|x_{i}-t_{i}\right|<\delta, i=1,2,3,4$, and $c<t_{1}<t_{2}<t_{3}<t_{4}<d$, and if $\left|y\left(x_{i}\right)-z_{i}\right|<\delta$, $i=1,2$, and $\left|y\left(x_{3}\right)-y\left(x_{4}\right)-z_{3}\right|<\delta$, then there exists a solution $z(x)$ of (1.1) satisfying $z\left(t_{i}\right)=z_{i}, i=1,2, z\left(t_{3}\right)-z\left(t_{4}\right)=z_{3}$, and $\left|y^{(i)}(x)-z^{(i)}(x)\right|<\epsilon$ on $[c, d], i=0,1,2$.

Proof. Fix a point $p_{0} \in(a, b)$, and define the set

$$
G=\left\{\left(x_{1}, x_{2}, x_{3}, x_{4}, C_{1}, C_{2}, C_{3}\right) \mid a<x_{1}<x_{2}<x_{3}<x_{4}<b, C_{1}, C_{2}, C_{3} \in \mathbb{R}\right\} .
$$

$G$ is an open subset of $\mathbb{R}^{7}$. Next, define a mapping $\phi: G \rightarrow \mathbb{R}^{7}$ by

$$
\phi\left(x_{1}, x_{2}, x_{3}, x_{4}, C_{1}, C_{2}, C_{3}\right)=\left(x_{1}, x_{2}, x_{3}, x_{4}, u\left(x_{1}\right), u\left(x_{2}\right), u\left(x_{3}\right)-u\left(x_{4}\right)\right),
$$

where $u(x)$ is the solution of (1.1) satisfying the initial conditions $u^{(i-1)}\left(p_{0}\right)=$ $C_{i}, i=1,2,3$. Condition (B) in Hypothesis 1.2 implies the continuity of solutions of initial value problems for (1.1) with respect to initial conditions, from whence we may derive the continuity of $\phi$. Moreover, the uniqueness assumption on solutions of (1.1):(1.2) implies that $\phi$ is one-one. It follows from the Brouwer theorem on invariance of domain [24, page 199] that $\phi(G)$ in an open subset of $\mathbb{R}^{7}$, and that $\phi$ is a homeomorphism from $G$ to $\phi(G)$. The statement of the theorem is then a direct result of the continuity of $\phi^{-1}$ and the fact that $\phi(G)$ is open.

We now establish that uniqueness of solutions for nonlocal 4-point problems implies uniqueness of solutions for nonlocal 3-point problems.

Theorem 2.3. Assume that solutions of (1.1):(1.2) are unique when they exist. Then, solutions of (1.1):(1.4) are unique, when they exist.

Proof. Assume for the purpose of contradiction that, for some $a<x_{1}<x_{2}<$ $x_{3}<b$ and $y_{1}, y_{2}, y_{3} \in \mathbb{R}$, there exist distinct solutions $y$ and $z$ of (1.1):(1.4); that is, $y\left(x_{1}\right)=z\left(x_{1}\right), y^{\prime}\left(x_{1}\right)=z^{\prime}\left(x_{1}\right), y\left(x_{2}\right)-y\left(x_{3}\right)=z\left(x_{2}\right)-z\left(x_{3}\right)$. By uniqueness of solutions of initial value problems for (1.1), we may assume, without loss of generality, that $y^{\prime \prime}\left(x_{1}\right)>z^{\prime \prime}\left(x_{1}\right)$.

Now fix $a<\tau<x_{1}$. By Theorem 2.2, for each $\epsilon>0$, there is a $\delta>0$ and there is a solution $z_{\delta}(x)$ of (1.1) such that

$$
z_{\delta}(\tau)=z(\tau), z_{\delta}\left(x_{1}\right)=z\left(x_{1}\right)+\delta, z_{\delta}\left(x_{2}\right)-z_{\delta}\left(x_{3}\right)=z\left(x_{2}\right)-z\left(x_{3}\right),
$$


and $\left|z_{\delta}(x)-z(x)\right|<\epsilon$ on $\left[\tau, x_{3}\right]$. For $\epsilon>0$, sufficiently small, there exist points $\tau<\tau_{1}<x_{1}<\tau_{2}<x_{2}$ so that

$$
z_{\delta}\left(\tau_{1}\right)=y\left(\tau_{1}\right), z_{\delta}\left(\tau_{2}\right)=y\left(\tau_{2}\right) .
$$

Also,

$$
z_{\delta}\left(x_{2}\right)-z_{\delta}\left(x_{3}\right)=z\left(x_{2}\right)-z\left(x_{3}\right)=y\left(x_{2}\right)-y\left(x_{3}\right),
$$

and by the hypotheses, $z_{\delta}(x) \equiv y(x)$. This is a contradiction. We conclude that solutions of (1.1):(1.4) are unique.

Of course, there is an analogue for the solutions of (1.1):(1.3) and (1.1):(1.5).

Theorem 2.4. Assume that solutions of (1.1):(1.3) are unique when they exist. Then, solutions of (1.1):(1.5) are unique when they exist.

The remainder of this section is devoted to a question converse to Theorems 2.3 and 2.4. In dealing with this converse question, we will make use of a lemma from [16] and 23] concerning a precompactness condition on bounded sequences of solutions of (1.1). The utility of this lemma in the context of our nonlocal boundary value problems arises from Remark 2.1(b) of this section.

Lemma 2.5. Assume the uniqueness of solutions for (1.1):(1.7) and (1.1):(1.8). If $\left\{y_{k}(x)\right\}$ is a sequence of solutions of (1.1) which is uniformly bounded on a nondegenerate compact subinterval $[c, d] \subset(a, b)$, then there is a subsequence $\left\{y_{k_{j}}(x)\right\}$ such that $\left\{y_{k_{j}}^{(i)}(x)\right\}$ converges uniformly on each compact subinterval of $(a, b)$, for each $i=0,1,2$.

Since uniqueness of solutions of (1.1):(1.4) and (1.1):(1.5) imply uniqueness of solutions of (1.1):(1.7) and (1.1):(1.8), we also have a statement of the the precompactness condition in terms of (1.1):(1.4) and (1.1):(1.5) .

Lemma 2.6. Assume the uniqueness of solutions for (1.1):(1.4) and (1.1):(1.5). If $\left\{y_{k}(x)\right\}$ is a sequence of solutions of (1.1) which is uniformly bounded on a nondegenerate compact subinterval $[c, d] \subset(a, b)$, then there is a subsequence $\left\{y_{k_{j}}(x)\right\}$ such that $\left\{y_{k_{j}}^{(i)}(x)\right\}$ converges uniformly on each compact subinterval of $(a, b)$, for each $i=0,1,2$.

Theorem 2.7. Assume that solutions of both (1.1):(1.4) and (1.1):(1.5) are unique when they exist. Then, solutions of both (1.1):(1.2) and (1.1):(1.3) are unique when they exist.

Proof. We will consider the uniqueness statement only for (1.1):(1.2), with the other argument being completely analogous. Assume to the contrary that, for some $a<x_{1}<x_{2}<x_{3}<x_{4}<b$ and $y_{1}, y_{2}, y_{3} \in \mathbb{R}$, there exist distinct solutions $y$ and $z$ of (1.1):(1.2).

By uniqueness of solutions of (1.1):(1.4), we may assume, with no loss of generality, that $y^{\prime}\left(x_{1}\right)<z^{\prime}\left(x_{1}\right)$ and $y^{\prime}\left(x_{2}\right)>z^{\prime}\left(x_{2}\right)$, and $y(x)<z(x)$ on $\left(x_{1}, x_{2}\right)$. In addition, by Remark 2.1(b) of this section, unique solutions exist for 2-point conjugate problems (1.1):(1.7) and (1.1):(1.8), as well as for the 3-point conjugate problem (1.1):(1.6). This implies $y(x)>z(x)$ on $(a, b) \backslash\left[x_{1}, x_{2}\right]$.

For each real $r \geq 0$, let $y_{r}(x)$ be the solution of (1.1):(1.7) satisfying the boundary conditions at $x_{3}$ and $x_{4}$ :

$$
y_{r}\left(x_{3}\right)=y\left(x_{3}\right), y_{r}^{\prime}\left(x_{3}\right)=y^{\prime}\left(x_{3}\right)-r, y_{r}\left(x_{4}\right)=y\left(x_{4}\right) .
$$


It follows from uniqueness of solutions of 3-point conjugate problems (1.1):(1.6) that, for $s>r \geqslant 0$,

$$
y(x) \leqslant y_{r}(x)<y_{s}(x) \text { on }\left(a, x_{3}\right)
$$

For each $r \geqslant 0$, let

$$
E_{r}=\left\{x_{1} \leq x \leq x_{2} \mid y_{r}(x) \leq z(x)\right\}
$$

and note that these sets are compact and nested such that $E_{s} \subset E_{r} \subset\left(x_{1}, x_{2}\right)$ when $s>r>0$.

For some $r>0$, suppose that $E_{r}=\emptyset$, and let $\eta$ be the least upper bound for those $s>0$ where $E_{s} \neq \emptyset$. Then, by continuity with respect to $r$ for solutions of the two point boundary value problem (1.1):(2.1), it follows that $E_{\eta} \neq \emptyset$; because, if $E_{\eta}=\emptyset$, then $E_{\eta-\epsilon}=\emptyset$ for sufficiently small $\epsilon>0$. Moreover, $y_{\eta}(x)=z(x)$ for every $x \in E_{\eta}$; because, if $y_{\eta}(x)<z(x)$ for some $x \in E_{\eta}$, then again by the continuity with respect to $r$ for solutions of (1.1):(2.1), $E_{\eta+\epsilon} \neq \emptyset$ for sufficiently small $\epsilon>0$.

By the uniqueness for solutions to the 3-point conjugate problem (1.1):(1.6), it follows that $E_{\eta}$ consists of at most two distinct points. If $\tau$ is one of these points, it necessarily follows that $y_{\eta}^{\prime}(\tau)=z^{\prime}(\tau)$. As a consequence of the hypotheses and the boundary conditions in (2.1), we see that

$$
y_{\eta}(\tau)=z(\tau), \quad y_{\eta}^{\prime}(\tau)=z^{\prime}(\tau), \quad y_{\eta}\left(x_{3}\right)-y_{\eta}\left(x_{4}\right)=z\left(x_{3}\right)-z\left(x_{4}\right),
$$

and hence that $y_{\eta}(x)=z(x)$, for all $x \in[a, b]$. This in turn implies, by the uniqueness of solutions to (1.1):(1.6), that $y(x)=z(x)$, for all $x \in[a, b]$ in violation of the of the initial assumption that $y(x)$ and $z(x)$ are different. Hence, $E_{n} \neq \emptyset$ for all $n \geqslant 1$, and

$$
\bigcap_{n=1}^{\infty} E_{n}:=E \neq \emptyset .
$$

Next, we observe that the set $E$ consists of a single point $x_{0}$ with $x_{1}<x_{0}<x_{2}$. In fact, if $t_{1}, t_{2} \in E$, with $x_{1}<t_{1}<t_{2}<x_{2}$, then the same type of argument that one uses to show the foregoing sets $E_{n}$ are nonnull leads to the conclusion that the interval $\left[t_{1}, t_{2}\right]$ must be contained in $E$. However, $\left[t_{1}, t_{2}\right] \subset E$ implies that the sequence $\left\{y_{n}(x)\right\}$ is uniformly bounded on $\left[t_{1}, t_{2}\right]$, which contradicts the part of Lemma 2.6 requiring boundedness for some subsequence of $\left\{y_{n_{j}}^{\prime}\left(x_{3}\right)\right\}$. Thus, we conclude $E=\left\{x_{0}\right\}$ with $x_{1}<x_{0}<x_{2}$, and

$$
\lim _{n \rightarrow \infty} y_{n}\left(x_{0}\right):=y_{0} \leq z\left(x_{0}\right) .
$$

Now we claim this is not possible. There are two cases to resolve. First assume $y_{0}=z\left(x_{0}\right)$. Then for $\epsilon>0$, sufficiently small, there is a solution $z(x, \epsilon)$ of the 3 -point conjugate boundary value problem for (1.1) satisfying

$$
z\left(x_{0}, \epsilon\right)=z\left(x_{0}\right)-\epsilon, z\left(x_{3}, \epsilon\right)=z\left(x_{3}\right), z\left(x_{4}, \epsilon\right)=z\left(x_{4}\right),
$$

and $z(x, \epsilon)<z(x)$ on $\left(a, x_{3}\right)$. Let us also note that

$$
z\left(x_{3}, \epsilon\right)-z\left(x_{4}, \epsilon\right)=z\left(x_{3}\right)-z\left(x_{4}\right)=y\left(x_{3}\right)-y\left(x_{4}\right) .
$$

Such a solution $z(x, \epsilon)$, where $\epsilon$ is chosen so that $z\left(x_{0}, \epsilon\right)=z\left(x_{0}\right)-\epsilon>y\left(x_{0}\right)$, can be used in place of $z(x)$ in defining the sets $\left\{E_{n}\right\}$ with respect to the given sequence of solutions $\left\{y_{n}(x)\right\}$. Then, as before, it would follow that each of these sets would be nonnull, which is impossible. 
For the remaining case, let $y\left(x_{0}\right)<y_{0}<z\left(x_{0}\right)$. In this case, for $0 \leq \lambda \leq 1$, let $z(x, \lambda)$ denote the solution of (1.1) satisfying the 2-point conjugate boundary conditions

$$
\begin{gathered}
z\left(x_{3}, \lambda\right)=\lambda y\left(x_{3}\right)+(1-\lambda) z\left(x_{3}\right), \\
z^{\prime}\left(x_{3}, \lambda\right)=\lambda y^{\prime}\left(x_{3}\right)+(1-\lambda) z^{\prime}\left(x_{3}\right), \\
z\left(x_{4}, \lambda\right)=\lambda y\left(x_{4}\right)+(1-\lambda) z\left(x_{4}\right) .
\end{gathered}
$$

We note that, for each $0 \leq \lambda \leq 1$,

$$
z\left(x_{3}, \lambda\right)-z\left(x_{4}, \lambda\right)=y\left(x_{3}\right)-y\left(x_{4}\right) .
$$

Let

$$
L=\left\{\left(z\left(x_{3}, \lambda\right), z^{\prime}\left(x_{3}, \lambda\right), z\left(x_{4}, \lambda\right)\right) \mid 0 \leq \lambda \leq 1\right\} .
$$

Then $L$ is a line segment in $\mathbb{R}^{3}$. The function $h:[0,1] \rightarrow L$ defined by

$$
h(\lambda)=\left(z\left(x_{3}, \lambda\right), z^{\prime}\left(x_{3}, \lambda\right), z\left(x_{4}, \lambda\right)\right)
$$

is continuous, one-one and onto. Next, define $g: L \rightarrow \mathbb{R}$ by

$$
g\left(\left(z\left(x_{3}, \lambda\right), z^{\prime}\left(x_{3}, \lambda\right), z\left(x_{4}, \lambda\right)\right)\right)=z\left(x_{0}, \lambda\right) .
$$

By continuous dependence of solutions on 2-point conjugate boundary conditions, $g$ is continuous and so $g \circ h:[0,1] \rightarrow \mathbb{R}$ is continuous. Now,

$$
g \circ h(0)=z\left(x_{0}\right)>y_{0}>y\left(x_{0}\right)=g \circ h(1) .
$$

Hence, there exists $0<\lambda_{0}<1$ so that

$$
g \circ h\left(\lambda_{0}\right)=z\left(x_{0}, \lambda_{0}\right)=y_{0} .
$$

Now, there is an $\eta>0$ such that $\left[x_{0}-\eta, x_{0}+\eta\right] \subset\left(x_{1}, x_{2}\right)$ and such that

$$
z\left(x, \lambda_{0}\right)<z(x) \text { on }\left[x_{0}-\eta, x_{0}+\eta\right] .
$$

Then with $\left\{y_{n}(x)\right\}$ the same sequence as before, we have

$$
\lim _{n \rightarrow \infty} y_{n}(x)>z(x)>z\left(x, \lambda_{0}\right),
$$

for all $x \in\left[x_{0}-\eta, x_{0}-\eta\right] \backslash\left\{x_{0}\right\}$, and

$$
\lim _{n \rightarrow \infty} y_{n}\left(x_{0}\right)=y_{0}=z\left(x_{0}, \lambda_{0}\right) .
$$

This is the same contradictory situation as in the case $y_{0}=z\left(x_{0}\right)$ considered previously.

From this final contradiction, we conclude that $y_{0} \leq z\left(x_{0}\right)$ is impossible, and therefore, solutions of (1.1):(1.2) are unique. Similarly, solutions of (1.1):(1.3) are unique.

\section{EXISTENCE OF SOLUTiOnS}

Having established, under the assumptions contained in Hypothesis 1.2, the equivalence of the uniqueness of solutions for both (1.1):(1.2) and (1.1):(1.3) with that of the uniqueness of solutions for both (1.1):(1.4) and (1.1):(1.5), we now deal with uniqueness implies existence for these problems. For the results of this section, continuous dependence as in Theorem 2.2 plays a role, as does the precompactness condition in Lemma 2.5. 
Theorem 3.1. Assume that solutions of (1.1):(1.2) are unique. Then, given a< $x_{1}<x_{2}<x_{3}<x_{4}<b$ and $y_{1}, y_{2}, y_{3} \in \mathbb{R}$, there exists a solution of (1.1):(1.2).

Proof. Let $a<x_{1}<x_{2}<x_{3}<x_{4}<b$ and $y_{1}, y_{2}, y_{3} \in \mathbb{R}$ be selected. We repeat again that 3-point, as well as 2-point, conjugate boundary value problems have unique solutions; that is, there exist unique solutions for each of (1.1):(1.6), (1.1):(1.7) and (1.1):(1.8). So, let $z(x)$ be the solution of (1.1) satisfying the 3-point boundary conditions at $x_{2}, x_{3}$ and $x_{4}$ :

$$
z\left(x_{2}\right)=y_{2}, z\left(x_{3}\right)=y_{3}, z\left(x_{4}\right)=0 .
$$

Next, define the set

$$
\begin{gathered}
S=\left\{u\left(x_{1}\right) \mid u \text { is a solution of (1.1) satisfying } u\left(x_{2}\right)=z\left(x_{2}\right),\right. \\
\text { and } \left.u\left(x_{3}\right)-u\left(x_{4}\right)=z\left(x_{3}\right)-z\left(x_{4}\right)\right\} .
\end{gathered}
$$

We observe first that $S$ is nonempty, since $z\left(x_{1}\right) \in S$.

Next, choose $s_{0} \in S$. Then, there is a solution $u_{0}(x)$ of (1.1) satisfying

$$
u_{0}\left(x_{1}\right)=s_{0}, u_{0}\left(x_{2}\right)=z\left(x_{2}\right), u_{0}\left(x_{3}\right)-u_{0}\left(x_{4}\right)=z\left(x_{3}\right)-z\left(x_{4}\right) .
$$

By Theorem 2.2. there exists a $\delta>0$ such that, for each $0 \leq\left|s-s_{0}\right|<\delta$, there is a solution $u_{s}(x)$ of (1.1) satisfying

$$
\begin{gathered}
u_{s}\left(x_{1}\right)=s, u_{s}\left(x_{2}\right)=u_{0}\left(x_{2}\right)=z\left(x_{2}\right), \text { and } \\
u_{s}\left(x_{3}\right)-u_{s}\left(x_{4}\right)=u_{0}\left(x_{3}\right)-u_{0}\left(x_{4}\right)=z\left(x_{3}\right)-z\left(x_{4}\right),
\end{gathered}
$$

or in other words, $s \in S$; in particular, $\left(s_{0}-\delta, s_{0}+\delta\right) \subset S$, and $S$ is an open subset of $\mathbb{R}$.

The remainder of the argument is devoted to showing that $S$ is also a closed subset of $\mathbb{R}$. To that end, we assume for the purpose of contradiction that $S$ is not closed. Then there exists an $r_{0} \in \bar{S} \backslash S$ and a strictly monotone sequence $\left\{r_{k}\right\} \subset S$ such that $\lim _{k \rightarrow \infty} r_{k}=r_{0}$.

We may assume, without loss of generality, that $r_{k} \uparrow r_{0}$. By the definition of $S$, we denote, for each $k \in \mathbb{N}$, by $u_{k}(x)$ the solution of (1.1) satisfying

$$
u_{k}\left(x_{1}\right)=r_{k}, u_{k}\left(x_{2}\right)=z\left(x_{2}\right) \text {, and } u_{k}\left(x_{3}\right)-u_{k}\left(x_{4}\right)=z\left(x_{3}\right)-z\left(x_{4}\right) .
$$

By uniqueness of solutions of (1.1):(1.2), we have

$$
u_{k}(x)<u_{k+1}(x) \text { on }\left(a, x_{2}\right) .
$$

Consequently, from Lemma 2.5 and the fact that $r_{0} \notin S$, we may conclude that $\left\{u_{k}(x)\right\}$ is not uniformly bounded above on each compact subinterval of each of $\left(a, x_{1}\right)$ and $\left(x_{1}, x_{2}\right)$.

Now, fix $a<\tau<x_{1}$ and let $w(x)$ be the solution of the 3-point conjugate boundary value problem for (1.1) satisfying

$$
w(\tau)=0, w\left(x_{1}\right)=r_{0}, w\left(x_{2}\right)=z\left(x_{2}\right) .
$$

It follows that, for some $K$ large, there exist points $a<\tau_{1}<x_{1}<\tau_{2}<x_{2}$ so that

$$
u_{K}\left(\tau_{1}\right)=w\left(\tau_{1}\right) \text { and } u_{K}\left(\tau_{2}\right)=w\left(\tau_{2}\right) .
$$

Also,

$$
u_{K}\left(x_{2}\right)=z\left(x_{2}\right)=w\left(x_{2}\right),
$$

which contradicts the uniqueness of solutions of the 3-point conjugate boundary value problem (1.1):(1.6). Thus, $S$ is also a closed subset of $\mathbb{R}$. 
In summary, $S$ is a nonempty subset of $\mathbb{R}$ that is both open and closed. We have $S \equiv \mathbb{R}$. By choosing $y_{1} \in S$, there is a corresponding solution $y(x)$ of (1.1) such that

$$
y\left(x_{1}\right)=y_{1}, y\left(x_{2}\right)=z\left(x_{2}\right)=y_{2}, y\left(x_{3}\right)-y\left(x_{4}\right)=z\left(x_{3}\right)-z\left(x_{4}\right)=y_{3} .
$$

There is, of course, an analogue of Theorem 3.1 with regard to uniqueness implies existence for solutions of (1.1):(1.3). In addition, our next result establishes that the hypotheses of Theorem 3.1 also yield the existence of solutions of the nonlocal problem (1.1):(1.4), and by analogy yields the result establishing the existence of solutions for the the nonlocal problem (1.1):(1.5).

Theorem 3.2. Assume the hypotheses of Theorem 3.1. Then, given a $<x_{1}<$ $x_{2}<x_{3}<b$ and $y_{1}, y_{2}, y_{3} \in \mathbb{R}$, there exists a unique solution of (1.1):(1.4).

Proof. Uniqueness of solutions of (1.1):(1.4) is from Theorem 2.3. So let $a<x_{1}<$ $x_{2}<x_{3}<b$ and $y_{1}, y_{2}, y_{3} \in \mathbb{R}$ be chosen. Also, fix $a<\tau<x_{1}$. Let $z(x)$ be the solution of the nonlocal boundary value problem (1.1):(1.2) obtained in Theorem 3.1 and satisfying

$$
z(\tau)=0, z\left(x_{1}\right)=y_{1}, z\left(x_{2}\right)-z\left(x_{3}\right)=y_{3} .
$$

This time, define the set

$$
\begin{gathered}
S=\left\{u^{\prime}\left(x_{1}\right) \mid u \text { is a solution of (1.1) satisfying } u\left(x_{1}\right)=z\left(x_{1}\right),\right. \\
\text { and } \left.u\left(x_{2}\right)-u\left(x_{3}\right)=z\left(x_{2}\right)-z\left(x_{3}\right)\right\} .
\end{gathered}
$$

Again $S$ is nonempty, and by arguments similar to those in proving Theorem 2.2, it follows from the uniqueness of solutions of (1.1):(1.4) that solutions depend continuously on such boundary conditions. It follows from this continuous dependence that $S$ is an open subset of $\mathbb{R}$.

As in the previous theorem, the remainder of the argument is devoted to showing $S$ is also a closed subset of $\mathbb{R}$. We assume, for contradiction, that $S$ is not closed. Then there is an $r_{0} \in \bar{S} \backslash S$ and a strictly monotone sequence $\left\{r_{k}\right\} \subset S$ such that $\lim _{k \rightarrow \infty} r_{k}=r_{0}$. Again, we may assume $r_{k} \uparrow r_{0}$.

By the definition of $S$, we denote, for each $k \in \mathbb{N}$, by $u_{k}(x)$ the solution of (1.1) satisfying

$$
u_{k}\left(x_{1}\right)=z\left(x_{1}\right), u_{k}^{\prime}\left(x_{1}\right)=r_{k}, \text { and } u_{k}\left(x_{2}\right)-u_{k}\left(x_{3}\right)=z\left(x_{2}\right)-z\left(x_{3}\right) .
$$

By uniqueness of solutions of (1.1):(1.2), we have

$$
u_{k}(x)>u_{k+1}(x) \text { on }\left(a, x_{1}\right) \text {, and } u_{k}(x)<u_{k+1}(x) \text { on }\left(x_{1}, x_{2}\right) .
$$

Lemma 2.5 and the fact that $r_{0} \notin S$ implies that $\left\{u_{k}(x)\right\}$ is not uniformly bounded below on each compact subinterval of $\left(a, x_{1}\right)$, and $\left\{u_{k}(x)\right\}$ is not uniformly bounded above on each compact subinterval of $\left(x_{1}, x_{2}\right)$.

Recalling that both 2-point and 3-point conjugate problems for (1.1) have unique solutions, let $w(x)$ be the solution of the 2-point conjugate boundary value problem for (1.1) satisfying

$$
w\left(x_{1}\right)=z\left(x_{1}\right), w^{\prime}\left(x_{1}\right)=r_{0}, w\left(x_{2}\right)=0 .
$$

It follows that, for some $K$ large, there exist points $a<\sigma_{1}<x_{1}<\sigma_{2}<x_{2}$ so that

$$
u_{K}\left(\sigma_{1}\right)=w\left(\sigma_{1}\right) \text { and } u_{K}\left(\sigma_{2}\right)=w\left(\sigma_{2}\right) .
$$


This time, we also have

$$
u_{K}\left(x_{1}\right)=z\left(x_{1}\right)=w\left(x_{1}\right),
$$

which contradicts the uniqueness of solutions of the 3-point conjugate boundary value problem (1.1):(1.6). Thus, $S$ is a closed subset of $\mathbb{R}$.

Again, $S \equiv \mathbb{R}$, and so we choose $y_{2} \in S$. We complete the proof by noting the presence of a solution, $y(x)$, of (1.1) such that

$$
y\left(x_{1}\right)=z\left(x_{1}\right)=y_{1}, y^{\prime}\left(x_{1}\right)=y_{2}, y\left(x_{2}\right)-y\left(x_{3}\right)=z\left(x_{2}\right)-z\left(x_{3}\right)=y_{3} .
$$

\section{Conclusions}

Established under the assumption of Hypothesis 1.2 the results contained in Sections 2 and 3 can now be summarized as follows:

(a) Uniqueness of solutions of (1.1):(1.2) and (1.1):(1.3) implies existence of solutions (1.1):(1.2) and (1.1):(1.3) .

(b) Solutions of both (1.1):(1.2) and (1.1):(1.3) are unique if, and only if, solutions of both (1.1):(1.4) and (1.1):(1.5) are unique.

(c) Uniqueness of solutions of (1.1):(1.4) and (1.1):(1.5) implies existence of solutions of (1.1):(1.4) and (1.1):(1.5).

\section{ACKNOWLEDGEMENTS}

The first author wishes to extend his thanks to Baylor University and its Department of Mathematics for their support and hospitality during the fall of 2004 when the research for this work was completed. Both authors wish to express their appreciation to the referee whose diligent reading and suggestions have helped to improve this paper.

\section{REFERENCES}

[1] C. Bai and J. Fang, Existence of multiple positive solutions for $m$-point boundary value problems, J. Math. Anal. Appl. 281 (2003), 76-85. MR.1980075 (2004b:34035)

[2] D. M. Goecke and J. Henderson, Uniqueness of solutions of right focal problems for third order differential equations, Nonlin. Anal. 8 (1984), 253-259. MR0738010 (85i:34006)

[3] C. P. Gupta and S. I. Trofimchuk, Solvability of a multi-point boundary value problem and a priori estimates, Canad. Appl. Math. Quart. 6 (1998), 45-60. MR1638415 (99f:34020)

[4] P. Hartman, Unrestricted n-parameter families, Rend. Circ. Mat. Palermo (2) 7 (1958), 123-142. MR0105470 (21:4211)

[5] P. Hartman, On $n$-parameter families and interpolation problems for nonlinear ordinary differential equations, Trans. Amer. Math. Soc. 154 (1971), 201-226. MR0301277 (46:435)

[6] J. Henderson, Uniqueness of solutions of right focal point boundary value problems for ordinary differential equations, J. Diff. Eqs. 41 (1981), 218-227. MR0630990 (83g:34018)

[7] J. Henderson, Existence of solutions of right focal point boundary value problems for ordinary differential equations, Nonlin. Anal. 5 (1981), 989-1002. MR0633013 (82j:34015)

[8] J. Henderson, Existence theorems for boundary value problems for $n t h$ order nonlinear difference equations, SIAM J. Math. Anal. 20 (1989), 468-478. MR0982673 (90a:39003)

[9] J. Henderson, Focal boundary value problems for nonlinear difference equations, I, J. Math. Anal. Appl. 141 (1989), 559-567. MR1009063 (90g:39003)

[10] J. Henderson, Focal boundary value problems for nonlinear difference equations, II, J. Math. Anal. Appl. 141 (1989), 568-579. MR1009063 (90g:39003)

[11] J. Henderson, Uniqueness implies existence for three-point boundary value problems for second order differential equations, Appl. Math. Lett. 18 (2005), 905-909. MR2152302 
[12] J. Henderson, B. Karna and C. C. Tisdell, Existence of solutions for three-point boundary value problems for second order equations, Proc. Amer. Math. Soc. 133 (2005), 1365-1369. MR2111960 (2005j:34026)

[13] J. Henderson and W. K. C. Yin, Existence of solutions for fourth order boundary value problems on a time scale, J. Differ. Eqs. Appl. 9 (2003), 15-28. MR.1958300(2003k:34041)

[14] L. K. Jackson, Uniqueness of solutions of boundary value problems for ordinary differential equations, SIAM J. Appl. Math. 24 (1973), 535-538. MR0322253 (48:615)

[15] L. K. Jackson, Existence and uniqueness of solutions of boundary value problems for third order differential equations, J. Diff. Eqs. 13 (1973), 432-437. MR0335925 (49:703)

[16] L. K. Jackson and K. Schrader, Existence and uniqueness of solutions of boundary value problems for third order differential equations, J. Diff. Eqs. 9 (1971), 46-54. MR0269920 (42:4813)

[17] G. Klaasen, Existence theorems for boundary value problems for $n t h$ order ordinary differential equations, Rocky Mtn. J. Math. 3 (1973), 457-472. MR0357944 (50:10409)

[18] A. Lasota and M. Luczynski, A note on the uniqueness of two point boundary value problems I, Zeszyty Naukowe UJ, Prace Matematyezne 12 (1968), 27-29. MR0224900 (37:499)

[19] A. Lasota and Z. Opial, On the existence and uniqueness of solutions of a boundary value problem for an ordinary second order differential equation, Colloq. Math. 18 (1967), 1-5. MR0219792 (36:2871)

[20] R. Ma, Existence theorems for a second-order three-point boundary value problem, J. Math. Anal. Appl. 212 (1997), 430-442. MR1464888 (98h:34041)

[21] R. Ma, Existence and uniqueness of solutions to first order three-point boundary value problems, Appl. Math. Lett. 15 (2002), 211-216. MR.1880760 (2002k:34032)

[22] A. C. Peterson, Existence-uniqueness for focal-point boundary value problems, SIAM J. Math. Anal. 12 (1982), 173-185. MR0605428 (83h:34017)

[23] K. Schrader, Uniqueness implies existence for solutions of nonlinear boundary value problems, Abstracts Amer. Math. Soc. 6 (1985), 235.

[24] E. H. Spanier, Algebraic Topology, McGraw-Hill, New York, 1966. MR.0210112 (35:1007)

Department of Mathematics, Baylor University, Waco, Texas 76798-7328

Current address: Department of Mathematics and Statistics, University of Missouri-Rolla, Rolla, Missouri 65409

E-mail address: sclark@umr.edu

URL: http://www.umr.edu/ ${ }^{\text {sclark }}$

Department of Mathematics, Baylor University Waco, Texas 76798-7328

E-mail address: JohnnyHenderson@baylor.edu

URL: http://www3.baylor.edu/ Johnny_Henderson/ 